\title{
Genome-wide DNA methylation analysis reveals hypomethylation in the low-CpG promoter regions in lymphoblastoid cell lines
}

\author{
Itsuki Taniguchi ${ }^{1}$, Chihiro Iwaya ${ }^{1}$, Keizo Ohnaka ${ }^{2}$, Hiroki Shibata ${ }^{1}$ and Ken Yamamoto ${ }^{3 *}$ (D)
}

\begin{abstract}
Background: Epidemiological studies of DNA methylation profiles may uncover the molecular mechanisms through which genetic and environmental factors contribute to the risk of multifactorial diseases. There are two types of commonly used DNA bioresources, peripheral blood cells (PBCs) and EBV-transformed lymphoblastoid cell lines (LCLs), which are available for genetic epidemiological studies. Therefore, to extend our knowledge of the difference in DNA methylation status between LCLs and PBCs is important in human population studies that use these DNA sources to elucidate the epigenetic risks for multifactorial diseases. We analyzed the methylation status of the autosomes for 192 and 92 DNA samples that were obtained from PBCs and LCLs, respectively, using a human methylation $450 \mathrm{~K}$ array. After excluding SNP-associated methylation sites and low-call sites, 400,240 sites were subjected to analysis using a generalized linear model with cell type, sex, and age as the independent variables.
\end{abstract}

Results: We found that the large proportion of sites showed lower methylation levels in LCLs compared with PBCs, which is consistent with previous reports. We also found that significantly different methylation sites tend to be located on the outside of the $\mathrm{CpG}$ island and in a region relatively far from the transcription start site. Additionally, we observed that the methylation change of the sites in the low-CpG promoter region was remarkable. Finally, it was shown that the correlation between the chronological age and ageing-associated methylation sites in ELOVL2 and FHL2 in the LCLs was weaker than that in the PBCs.

Conclusions: The methylation levels of highly methylated sites of the low-CpG-density promoters in PBCs decreased in the LCLs, suggesting that the methylation sites located in low-CpG-density promoters could be sensitive to demethylation in LCLs. Despite being generated from a single cell type, LCLs may not always be a proxy for DNA from PBCs in studies of epigenome-wide analysis attempting to elucidate the role of epigenetic change in disease risks.

Keywords: DNA methylation, Lymphoblastoid cell lines, Epigenome-wide analysis, Epigenetic epidemiology, Human methylation array

\section{Background}

The DNA obtained from EBV-transformed immortalized lymphoblastoid cell lines (LCLs) and peripheral blood cells (PBCs) are commonly used in medical genetic studies. LCLs can be generated from both healthy individuals and patients and supply an unlimited source of genomic

\footnotetext{
* Correspondence: yamamoto_ken@med.kurume-u.ac.jp

${ }^{3}$ Department of Medical Biochemistry, Kurume University School of Medicine, 67 Asahi-machi, Kurume, Fukuoka 830-0011, Japan

Full list of author information is available at the end of the article
}

DNA. Additionally, LCLs and PBCs have been successfully used for gene expression analyses [1].

DNA methylation is one of the important epigenetic mechanisms regulating gene expression. In addition to sequence variants, it is increasingly accepted that this DNA modification may be implicated in the susceptibility of various multifactorial diseases [2-4]. Recent developments in technology for human genome analysis have enabled us to identify disease-related DNA methylation changes at the genome-wide level. Because it is essential 
to use relatively large samples in searching for genes that are susceptible to multifactorial diseases, the DNA sources are limited to LCLs, PBCs, and saliva. However, it is known that DNA methylation status varies between cell types [5]. Therefore, to extend our knowledge of the difference in DNA methylation status between LCLs and PBCs is important in human population studies that use these DNA sources to elucidate the epigenetic risks for multifactorial diseases.

To this end, we designed experiments to compare the DNA methylation status between LCLs and PBCs at an epigenome-wide level using approximately 400,000 methylation data sites from 92 LCL and 192 PBC samples obtained using the Human Methylation $450 \mathrm{~K}$ array. We analyzed global differences in methylation profiles and the degree of difference in methylation level of each site in terms of location (inside or outside the CpG island, the distance from transcription start site and promoter type) between LCLs and PBCs. Additionally, the association strength of methylation levels at the ageing-related methylation sites in FHL2 and ELOVL2 with chronological age was compared between LCLs and PBCs.

\section{Methods}

\section{Subjects}

EBV-transformed LCLs derived from 92 healthy Japanese subjects were provided by the Riken Bioresource Center Cell Bank [6]. PBCs were obtained from 192 participants of a baseline survey of the general population from a Fukuoka-based cohort study [7, 8]. This study was performed in accordance with the principles of the Declaration of Helsinki and was approved by the Institutional Review Board at Kyushu University.

\section{DNA methylation chip assay}

Genomic DNA was bisulfite-treated using the EZ-96 DNA Methylation Kit (Zymo Research Corporation, Orange, CA), which combines bisulfite conversion and DNA cleanup in a 96-well plate. Genome-wide DNA methylation profiles were obtained using the Illumina HumanMethylation450 BeadChip (Illumina, San Diego, CA) according to the manufacturer's instructions. The GenomeStudio V2011.1 (Methylation Module version 1.9.0) was employed to determine the beta values that reflected the estimated methylation level for each CpG site. The beta value was calculated as: $\operatorname{Max}(-$ signal for methylation, 0)/[Max(signal for methylation, $0)+\operatorname{Max}($ signal for unmethylation, 0)+100]. Using this metric, the DNA methylation level was represented by a number between 0 (no methylation) and 1 (complete methylation). The signal intensities were normalized to the internal controls and background prior to beta value calculation.

\section{Selection and classification of DNA methylation sites}

Among 473,864 methylation sites on the autosomes, 1305 sites showing low calls $(<0.95)$ were removed for further analyses. To eliminate SNP-associated methylation sites, we screened the nearest SNP for each methylation site using the dbSNP135 database (SNPs categorized in weight = 1 group, http://www.ncbi.nlm.nih.gov/SNP/). We found 72,318 sites in which SNPs were located on the $\mathrm{C}$ or $\mathrm{G}$ site. Additionally, one methylation site demonstrated an outlier value. After removing these sites; 400,240 methylation sites on the array were available for further analyses. Based on the CpG Islands (CGI) track of the UCSC table browser of the UCSC Genome Bioinformatics database (http://genome.ucsc.edu/index.html), the 400,240 sites on autosomes were classified into two groups, CGI-sites $(135,674$ sites, inside of CGI) or nonCGI-sites (264,566 sites, outside the CGI). Among the non-CGI sites, 95,625 sites were located near CGI $( \pm 2,000$ bases) that were classified in a shore group. The distance between the methylation site and the nearest transcription start site (TSS) was calculated using the NCBI RefSeq database. The physical positions on the human genome were based on the Genome Reference Consortium Human Build 37 (GRCh37, http://www.ncbi.nlm.nih.gov/ assembly/). Of 400,240 probes, 159,688 demonstrated a TSS between -500 bases and $+2,000$ bases; among these, 85,700 sites could be classified into high-CpG-density promoters (HCP), intermediate-CpG-density promoters (ICP) and low-CpG-density promoters (LCP), as reported by Mikkelsen et al. [9] $(69,836,10,719$, and 5145 in HCP, ICP, and LCP, respectively).

\section{Statistical analysis}

To evaluate the difference in methylation level of each site, the data were analyzed using modeling individual Illumina beta values using a generalized linear model (glm) with cell type (LCLs or PBCs), age and sex as the independent variables. $P$ values and the difference in methylation level for each cell type were obtained. The statistical power to detect methylation differences of 0.25 and 0.5 between 192 PBCs and 92 LCLs was estimated to be 50.2 and $97.5 \%$, respectively at a significance level of $P=0.05$ using $G *$ Power 3.1 software [10]. A principal component analysis (PCA) was performed using the beta values for the 400,240 sites, and the first and second principal component scores for each sample were plotted. The regression analysis was performed using the chronological age of the subjects and the beta values of cg06639320 and cg16867657 for FHL2 and ELOVL2, respectively, with adjustments for sex. These analyses were performed using $\mathrm{R}$ (release 2.15.2). 


\section{Results}

Comparison of global DNA methylation profiles between LCLs and PBCs

To assess the global difference of DNA methylation levels between LCLs and PBCs, we performed a PCA using the methylation data of 400,240 sites on autosomes obtained using the $450 \mathrm{~K}$ methylation array. As shown in Fig. 1a, the LCL and PBC groups were clearly distinguished by their first principal component score. Additionally, the PBC samples were distributed within a narrow range, whereas the LCL samples showed a relatively wide range in the second principal component score. These results suggest that there is a global difference in DNA methylation levels between these cell types and that the levels are more diverse in LCLs than in PBCs.

We then examined the difference in methylation level for each site using a glm adjusted for age and sex. As shown in the volcano plot in Fig. 1b, the sites showing lower levels in LCL than in PBC were predominant (low-met-LCL group). The 138,871 sites (34.7\% of the total) showed $-\log _{10}(P$ value $)>10$; among these sites, $85.1 \%$ were in the low-met-LCL group. This inclination was observed in each autosome (Additional file 1: Figure $\mathrm{S} 1$ ). Therefore, it was suggested that the main difference in DNA methylation between LCLs and PBCs was hypomethylation in the LCLs and that the change in methylation levels occurred globally in the autosomes.

\section{Hypomethylation observed in the LCLs occurs at sites outside the GpG island}

We next assessed the distribution of the difference in methylation levels between LCLs and PBLs in terms of the location of the site (inside or outside the CpG island) (named CGI-site or non-CGI-site). As shown in Fig. 2a, the distribution of difference was dissimilar between them; the proportion of the sites showing a low $P$ value was larger in the non-CGI-site group (black solid line) than in the CGI-site group (black dashed line). This trend was apparent in the low-met-LCL group (compare the red solid and dashed lines), whereas a dissimilarity of distribution was not observed in the high-met-LCL group (compare the blue solid and dashed lines). These results prompted us to further classify the non-CGI-sites into shore or non-shore groups because the CGI shores were suggested to contribute to tissue-specific DNA methylation $[11,12]$. However, we did not find significant differences in the distribution between the shore and non-shore group of the low-met-LCL (Fig. 2b). Taken together, these results suggested that the majority of hypomethylation observed in the LCLs occurred at sites outside the CGIs regardless of shores.

\section{Comparison of the difference in DNA methylation levels observed among LCLs and PBCs in terms of distance from the transcription start site}

We further examined the relationship between the distance from the TSS and the difference in DNA methylation levels observed among LCLs and PBCs. We plotted $-\log _{10}(P$ value $)$ for each site against the distance from the nearest TSS (shown in gray dots in Fig. 3a) and indicated a proportion of the site showing $\log _{10}(P$ value $)>10,25$, and 50 in blue, green, and pink dots, respectively (Fig. 3a). The proportion was calculated by dividing the number of the sites meeting the $P$ value criteria by the total number of sites within \pm 50 bases of window size. We found that the proportion of significantly different sites was lower near the TSS. For instance, approximately $25 \%$ of the sites near the TSS
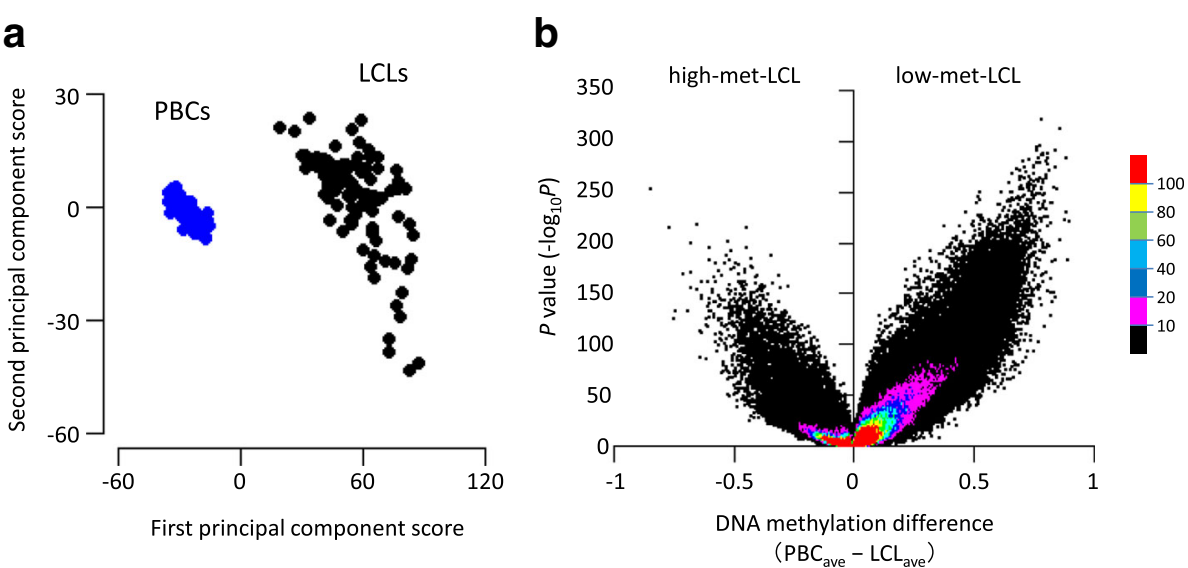

Fig. 1 Global difference in the DNA methylation level between the LCLs and PBCs. a Principal component analysis (PCA) plot. PCA was performed using the methylation level of the 400,240 sites on autosomes. The LCL and PBC samples are shown in black and blue dots, respectively. b Volcano plot with the difference of the average of DNA methylation level on the $x$-axis and the $P$ value $\left(-\log _{10} P\right)$ obtained via glm analysis on the $y$-axis. Each color shows the dot density $(100<n, 80<n \leq 100,60<n \leq 80,40<n \leq 60,20<n \leq 40,10<n \leq 20$ and $n \leq 10$ per unit area $(0.002 \times 1$ for $x$ and $y$-axis, respectively) in red, yellow, green, sky blue, blue, pink, and black, respectively) 

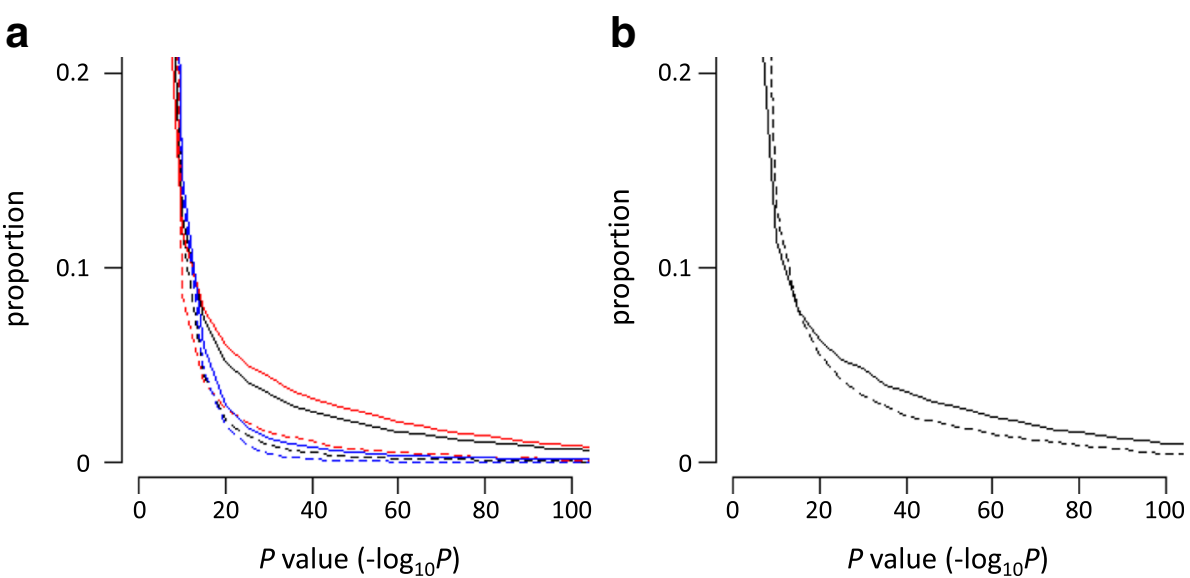

Fig. 2 Distribution of the differences in methylation levels between LCLs and PBLs in terms of CGI. a The proportion of $P$ values obtained from non-CGI and CGI sites in all samples (black solid and dashed lines, respectively), non-CGl and CGI sites in the low-met-LCL group (red solid and dashed lines, respectively), and non-CGI and CGI sites in the high-met-LCL group (blue solid and dashed lines, respectively) are indicated. $\mathbf{b}$ The proportion of $P$ values obtained from the non-shore and shore sites (solid and dashed lines, respectively) in the non-CGI sites of the low-met-LCL group are indicated

showed $-\log _{10}(P$ value $)>10$, whereas this proportion increased to approximately $45 \%$ for the sites located approximately \pm 1000 bases from the TSS in the lowmet-LCL group (blue dots, left panel of Fig. 3a). This trend was also observed even in the lower $P$ value threshold group (green and pink dots) and in the high-met-LCL group (right panel of Fig. 3a). We then analyzed the sites showing $-\log _{10}(P$-value $)>10$ separately for CGI- and non-CGI-site groups. As shown in Fig. $3 \mathrm{~b}$, the proportion of non-CGI-sites near the TSS was high in both the low- and high-met-LCL groups (red and blue dots, respectively, Fig. 3b). However, the lowest proportion was observed near the TSS in the case of CGI-sites (pink and sky blue dots for low- and highmet-LCL groups, respectively, Fig. 3b). These results suggested that the low CpG promoter would show a more significant difference in DNA methylation levels than the high $\mathrm{CpG}$ promoter.

\section{The methylation sites located in low CpG promoters could be sensitive to demethylation in LCLs}

To assess whether the promoter type affects the difference in DNA methylation levels between LCLs and PBCs, the methylation sites located in HCP, LCP and ICP were extracted based on the data set of Mikkelsen et al. [9] $(69,836,10,719$, and 5,145, in HCP, ICP, and LCP, respectively), and analyzed the distribution of $\log _{10}(P$ value $)$ in all, low- and high-met-LCL groups (Fig. 4). It was shown that the proportion of differentially methylated sites was higher in the LCPs than the HCPs.
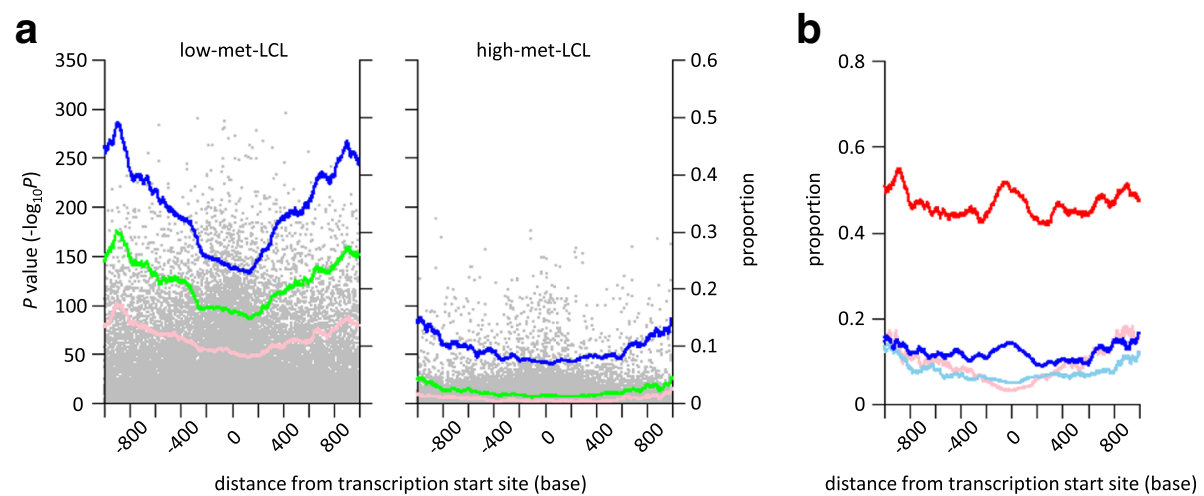

Fig. 3 Distribution of the differences in methylation levels between LCLs and PBLs in terms of TSS. a $P$ values were plotted against the distance from the nearest TSS (gray dots). The proportion of the sites with $P$ values $\left(-\log _{10} P\right)$ greater than 10 (blue dots), 25 (green dots), and 50 (pink dots) in a window size of \pm 50 bases were plotted. $\mathbf{b}$ The proportion of the sites with $P$ values $\left(-\log _{10} P\right)$ greater than 10 obtained from non-CGl and CGI sites in the low-met-LCL group (red and pink dots, respectively), and from non-CGl and CGI sites in the high-met-LCL group (blue and sky blue dots, respectively) in a window size of \pm 50 bases were plotted against TSS 
ALL

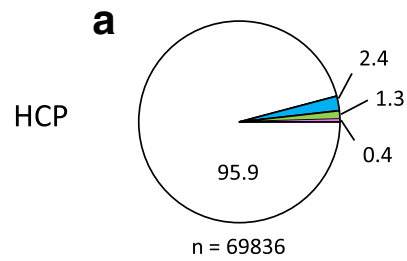

ICP

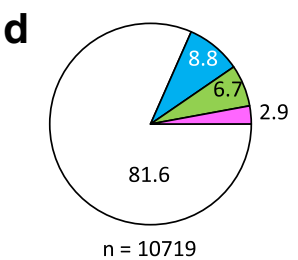

LCP

g

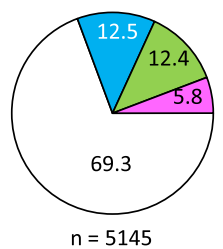

low-met-LCL

b

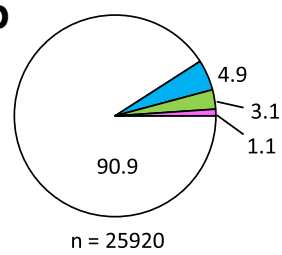

e

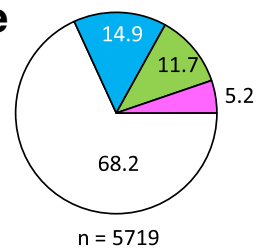

h

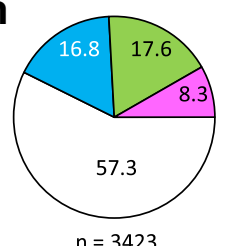

high-met-LCL

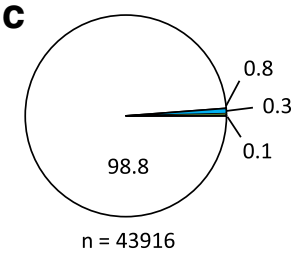

f
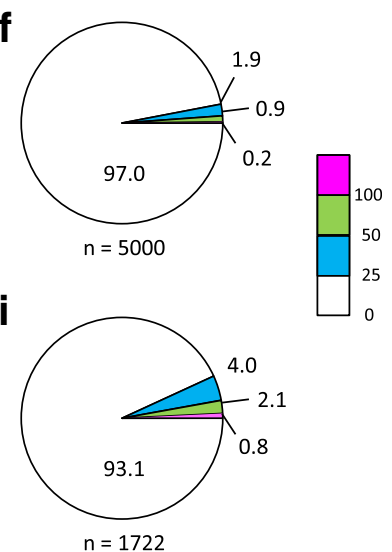

Fig. 4 Difference in methylation levels between LCLs and PBLs in terms of promoter type. The proportion of the sites with $P$ values $\left(-\log _{10} P\right) \leq$ 25, $25 \sim 50,50 \sim 100$, and $\geq 100$ are indicated in white, blue, green, and red, respectively. The results obtained from the HCP, ICP, and LCP sites in all samples (a, d, and $\mathbf{g}$, respectively) in the low-met-LCL group (b, e, and $\mathbf{h}$, respectively) and in the high-met-LCL (c, $\mathbf{f}$, and $\mathbf{i}$, respectively) are shown

In the LCPs, the proportion of the sites showing$\log _{10}(P$ value $)>25$ was $30.7 \%$, whereas that in HCPs was $4.1 \%$ in all sites (compare Fig. 4a, g). This was more pronounced in the low-met-LCL group (compare Fig. 4b, c, h, i). The sites located in ICPs showed intermediate values between HCPs and LCPs (Fig. 4d-f). These results suggested that the methylation sites located in low CpG promoters could be sensitive to demethylation in LCLs.

To further assess promoter type differences, we compared the HCPs and LCPs methylation level profiles. As shown in Fig. 5, nearly half of the sites in LCPs showed more than 0.6 methylation levels, whereas almost all sites in HCPs were hypomethylated in PBCs. Additionally, it was observed that the methylation levels of highly methylated sites of the LCPs decreased in the LCLs. Therefore, we concluded that highly methylated sites of LCPs caused the difference in DNA methylation levels observed between HCPs and LCPs, especially in the lowmet-LCL group.

\section{Comparison between LCLs and PBCs regarding the association between ageing-related CpG sites and chronological age}

Using DNA obtained from PBCs, it has been reported that the methylation levels of several $\mathrm{CpG}$ sites are associated with chronological age. However, it remains unclear whether LCLs should be utilized for studies on epigenetic ageing biomarkers. To address this issue, we performed a regression analysis for chronological age and known ageing-related $\mathrm{CpG}$ sites located in FHL2 and ELOVL2 [13, 14]. FHL2 encodes a member of the four-and-a-half-LIM-only protein family which is suggested to have a role in the assembly of extracellular membranes and in transformation of normal myoblasts to rhabdomyosarcoma cells (OMIN 602633). ELOVL2 encodes an enzyme which catalyzes the first and ratelimiting reaction of the long-chain fatty acids elongation cycle (OMIM 611814). As shown in Fig. 6a, the methylation level of the PBCs was highly correlated with chronological age (blue dots, $P=1.7 \mathrm{E}-18$ and $r^{2}=0.33$ for FHL2, $P=3.1 \mathrm{E}-25$ and $r^{2}=0.44$ for ELOVL2). In contrast, the methylation level of the LCLs was varied and the association was weak (black dots, $P=0.04$ and $r^{2}=0.05$ for FHL2, $P=1.9 \mathrm{E}-5$ and $r^{2}=0.18$ for ELOVL2). Therefore, these results suggest that DNA obtained from LCLs may not always be an alternative to DNA from PBCs.

\section{Discussion}

In this study, we used a $450 \mathrm{~K}$ methylation array to investigate the methylation differences between LCLs and PBCs, which are commonly used in genetic epidemiological 
LCP

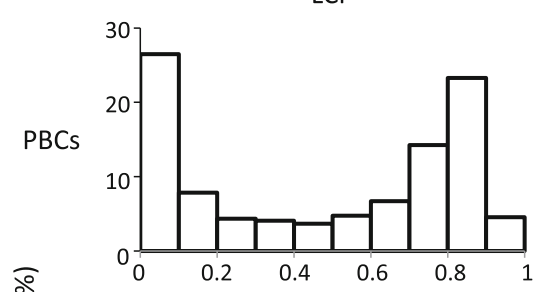

$\frac{0}{0}$
$\frac{0}{0}$
$\frac{0}{0}$
$\frac{0}{2}$

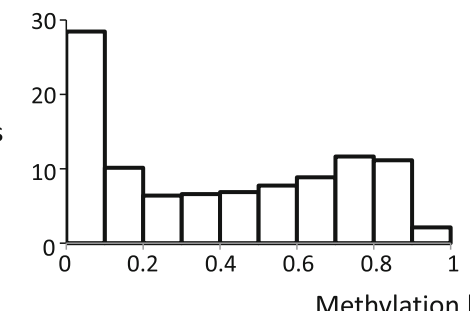

HCP
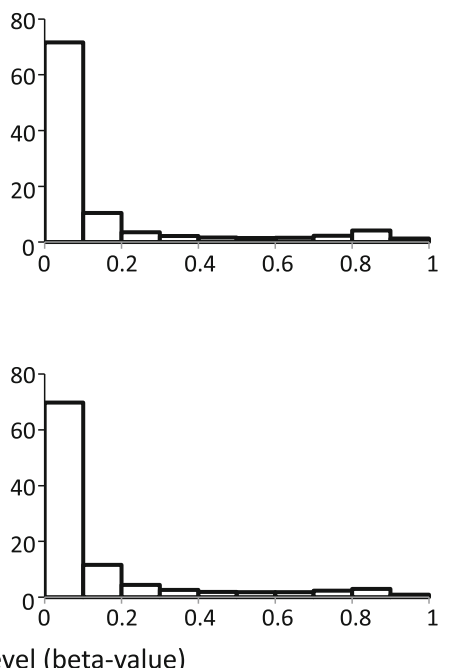

Fig. 5 Distribution of the methylation levels of the sites in the LCPs and HCPs. The frequency in the LCLs and PBCs are shown separately

studies. In all genomes, the majority of the sites in the LCLs showed lower methylation levels than those of the PBCs, and these sites were primarily located in non-CGI regions. Additionally, we found that differentially methylated sites were predominantly located in the LCP region.

Although a relatively small sample number and number of methylation sites were analyzed, previous studies showed that methylation status in LCLs is different from that of PBCs and that the methylation level in LCLs is lower than that of PBCs in the majority of sites [15-20].
Because a large number of samples and more sites were examined, we could investigate the differences in methylation levels between LCLs and PBCs in terms of CGI location, distance from TSS and promoter type as characterized by CG density. We found that a fraction showing a significant difference in methylation level between the LCLs and PBCs was observed near the TSS in the non-CGI sites but not in the CGI sites. This result suggests that the difference in the methylation level of these cell types would be high in the genes in which the promoter shows a low GC content.
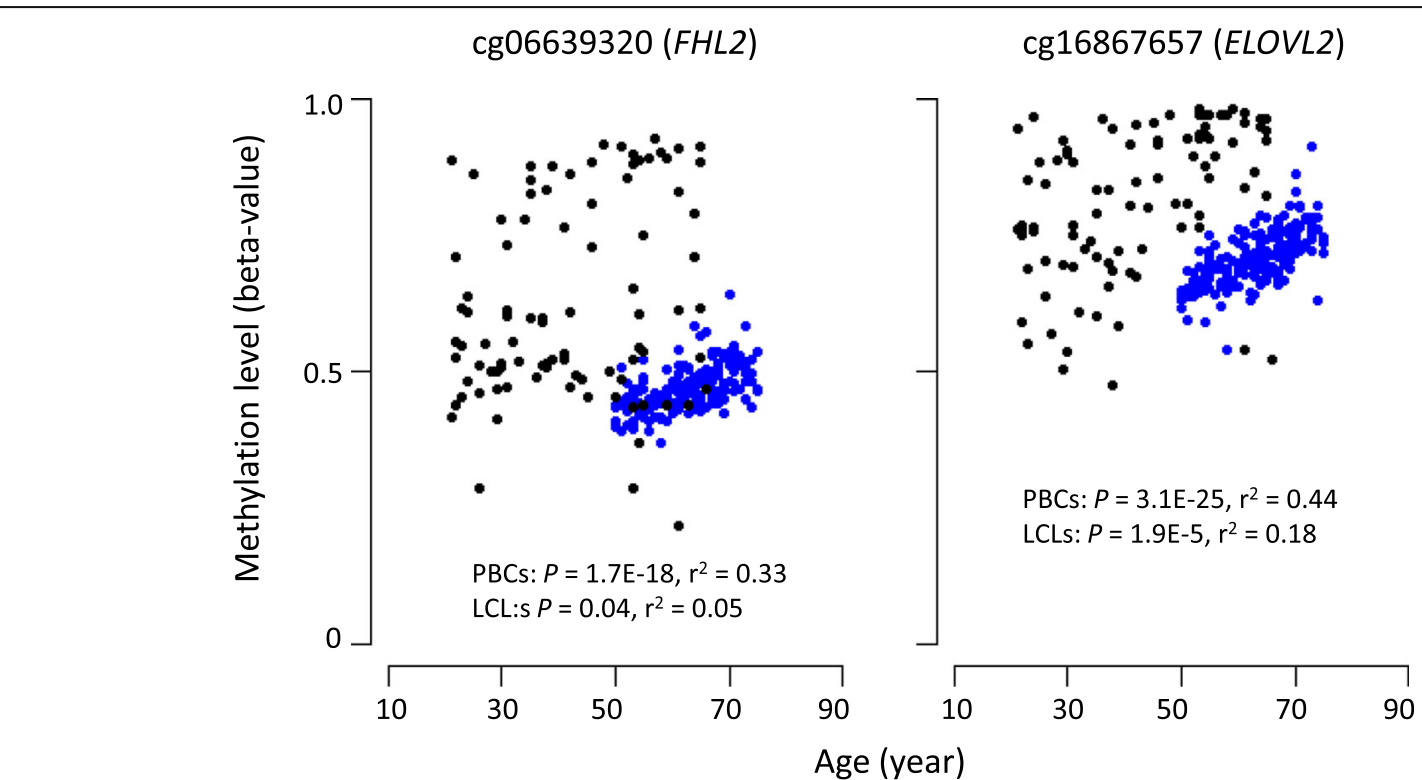

Fig. 6 Regression analyses of the methylation levels and chronological age at the FHL2 and ELOVL2 loci. The methylation levels in the LCLs (black dots) and PBCs (blue dots) were plotted against the age of the donors at the time of providing the specimens. The $P$ values and $r^{2}$ were obtained by correcting for sex 
We found that significantly different methylation sites were predominant in LCPs but not in HCPs. It has been demonstrated that LCPs are generally associated with tissue-specific genes, whereas HCPs are associated with two classes of genes, including ubiquitous "housekeeping" genes and highly regulated "key developmental" genes $[9,21,22]$. Therefore, our results suggest that the methylation sites located in promoters classified as LCP could have a functional role in distinguishing between LCLs and PBCs by regulating the corresponding gene expression.

The epigenome-wide association studies using human population samples to identify the disease risk loci and epigenomes that are affected by intrinsic or extrinsic factors, such as ageing and smoking, have been progressing $[13,14,23,24]$. We evaluated the differences in association strength between well-known ageing methylation sites and the chronological age of the samples between LCLs and PBCs and found that the correlation was more significant in PBCs than LCLs. This was due to a larger variance of methylation levels in LCLs than in PBCs. In addition to the differences in cell type, artificial experimental processes, including in vitro culture, culture period and culture freezing, and thawing could cause the large variances in data observed in the LCLs. Therefore, we concluded that DNA obtained from LCLs may not always be a proxy for DNA from PBCs in studies of epigenome-wide analysis attempting to elucidate the role of epigenetic change in disease risks.

\section{Conclusion}

There is a global difference in DNA methylation levels between LCLs and PBCs, and the main difference was hypomethylation in the LCLs. The methylation levels of highly methylated sites of the low-CpG-density promoters in PBCs decreased in the LCLs, suggesting that the methylation sites located in low-CpG-density promoters could be sensitive to demethylation in LCLs. The correlation between well-known ageing methylation sites and the chronological age of the samples was more significant in PBCs than LCLs, indicating that despite being generated from a single cell type, LCLs may not always be a proxy for DNA from PBCs in studies of epigenomewide analysis attempting to elucidate the role of epigenetic change in disease risks.

\section{Additional file}

Additional file 1: Figure S1. Volcano plot for each autosome with the difference of the average of the DNA methylation levels on the $x$-axis and the $P$ value $\left(-\log _{10} P\right)$ obtained via $g l m$ analysis on the $y$-axis. Each color shows the dot density $(100<n, 80<n \leq 100,60<n \leq 80,40<n \leq$ $60,20<n \leq 40,10<n \leq 20$, and $n \leq 10$ per unit area $(0.002 \times 1$ for the $x$-axis and $y$-axis, respectively) in red, yellow, green, sky blue, blue, pink and black, respectively). (PDF $295 \mathrm{~kb}$ )

\section{Abbreviations}

CGl: CpG Island; ELOVL2: Elongation of very long chain fatty acids protein 2; FHL2: Four and a half LIM domains 2; GIm: Generalized linear model; HCP: High-CpG-density promoter; ICP: Intermediate-CpG-density promoter; LCL: Lymphoblastoid cell line; LCP: Low-CpG-density promoter; PBC: Peripheral blood cell; PCA: Principal component analysis;

TSS: Transcription start site

\section{Acknowledgements}

We thank all of the people who have continuously supported the population-based cohort study, the Kyushu University Fukuoka Cohort Study. We also thank Ms. Miki Sonoda for her technical assistance.

\section{Funding}

This work was supported by KAKENHI Grant Number 15 K08290 from the Japan Society for the Promotion of Science.

\section{Availability of data and materials}

The datasets used and/or analyzed during the current study are available from the corresponding author on reasonable request.

\section{Authors' contributions}

$\mathrm{KO}$ performed the sample collection KO. KY performed the DNA methylation chip experiments. IT, $\mathrm{Cl}$, and $\mathrm{KY}$ performed the statistical and bioinformatics analyses. HS supervised the research. All authors wrote and approved the manuscript.

\section{Competing interests}

The authors declare that they have no competing interests.

\section{Consent for publication}

Not applicable.

\section{Ethics approval and consent to participate}

The study protocol was approved by the local ethics committee (Kyushu University Regional and Institutional Committee of Science and Research Ethics; Number 481-03), and written informed consent was provided by all participants.

\section{Publisher's Note}

Springer Nature remains neutral with regard to jurisdictional claims in published maps and institutional affiliations.

\section{Author details}

${ }^{1}$ Division of Genomics, Medical Institute of Bioregulation, Kyushu University, 3-1-1 Maidashi, Higashi-ku, Fukuoka 812-8582, Japan. ²Department of Geriatric Medicine, Graduate School of Medical Sciences, Kyushu University, 3-1-1 Maidashi, Higashi-ku, Fukuoka 812-8582, Japan. ${ }^{3}$ Department of Medical Biochemistry, Kurume University School of Medicine, 67 Asahi-machi, Kurume, Fukuoka 830-0011, Japan.

Received: 2 March 2017 Accepted: 4 May 2017

Published online: 12 May 2017

\section{References}

1. Powell JE, Henders AK, McRae AF, Wright MJ, Martin NG, Dermitzakis ET, Montgomery GW, Visscher PM. Genetic control of gene expression in whole blood and lymphoblastoid cell lines is largely independent. Genome Res. 2012;22:456-66.

2. Ordovás JM, Smith CE. Epigenetics and cardiovascular disease. Nat Rev Cardiol. 2010:7:510-19.

3. Costenbader KH, Gay S, Alarcón-Riquelme ME, laccarino L, Doria A. Genes, epigenetic regulation and environmental factors: which is the most relevant in developing autoimmune diseases? Autoimmun Rev. 2012;11:604-9.

4. Keating ST, El-Osta A. Epigenetic changes in diabetes. Clin Genet. 2013;84:1-10.

5. Ziller MJ, Gu H, Müller F, Donaghey J, Tsai LT, Kohlbacher O, De Jager PL, Rosen ED, Bennett DA, Bernstein BE, et al. Charting a dynamic DNA methylation landscape of the human genome. Nature. 2013;500:477-81.

6. Iwakawa M, Goto M, Noda S, Sagara M, Yamada S, Yamamoto N, Kawakami Y, Matsui $Y$, Miyazawa $Y$, Yamazaki $H$, et al. DNA repair capacity measured by high throughput alkaline comet assays in EBV-transformed cell lines and peripheral blood cells from cancer patients and healthy volunteers. Mutat Res. 2005;588:1-6. 
7. Nanri A, Yoshida D, Yamaji T, Mizoue T, Takayanagi R, Kono S. Dietary patterns and C-reactive protein in Japanese men and women. Am J Clin Nutr. 2008;87:1488-96.

8. Yoshida D, Toyomura K, Fukumoto J, Ueda N, Ohnaka K, Adachi M, Takayanagi R, Kono S. Waist circumference and cardiovascular risk factors in Japanese men and women. J Atheroscler Thromb. 2009;16:431-41.

9. Mikkelsen TS, Ku M, Jaffe DB, Issac B, Lieberman E, Giannoukos G, Alvarez P, Brockman W, Kim TK, Koche RP, et al. Genome-wide maps of chromatin state in pluripotent and lineage-committed cells. Nature. 2007;448:553-60.

10. Faul F, Erdfelder E, Lang AG, Buchner A. G*Power 3: a flexible statistical power analysis program for the social, behavioral, and biomedical sciences. Behav Res Methods. 2007;39:175-91.

11. Irizarry RA, Ladd-Acosta C, Wen B, Wu Z, Montano C, Onyango P, Cui H, Gabo K, Rongione M, Webster M, et al. The human colon cancer methylome shows similar hypo- and hypermethylation at conserved tissuespecific CpG island shores. Nat Genet. 2009;41:178-86.

12. Doi A, Park IH, Wen B, Murakami P, Aryee MJ, Irizarry R, Herb B, Ladd-Acosta C, Rho J, Loewer S, et al. Differential methylation of tissue- and cancerspecific CpG island shores distinguishes human induced pluripotent stem cells, embryonic stem cells and fibroblasts. Nat Genet. 2009;41:1350-53.

13. Garagnani P, Bacalini MG, Pirazzini C, Gori D, Giuliani C, Mari D, Di Blasio AM, Gentilini D, Vitale G, Collino S, et al. Methylation of ELOVL2 gene as a new epigenetic marker of age. Aging Cell. 2012;11:1132-4.

14. Hannum G, Guinney J, Zhao L, Zhang L, Hughes G, Sadda S, Klotzle B, Bibikova M, Fan JB, Gao Y, et al. Genome-wide methylation profiles reveal quantitative views of human aging rates. Mol Cell. 2013:49:359-67.

15. Brennan EP, Ehrich M, Brazil DP, Crean JK, Murphy M, Sadlier DM, Martin F, Godson C, McKnight AJ, van den Boom D, et al. Comparative analysis of DNA methylation profiles in peripheral blood leukocytes versus lymphoblastoid cell lines. Epigenetics. 2009;:159-64.

16. Sun $\mathrm{Y}$, Turner ST, Smith JA, Hammond PI, Lazarus A, Van De Rostyne JL, Cunningham JM, Kardia SL. Comparison of the DNA methylation profiles of human peripheral blood cells and transformed B-lymphocytes. Hum Genet. 2010;127:651-8.

17. Grafodatskaya D, Choufani S, Ferreira JC, Butcher DT, Lou Y, Zhao C, Scherer SW, Weksberg R. EBV transformation and cell culturing destabilizes DNA methylation in human lymphoblastoid cell lines. Genomics. 2010;95:73-83.

18. Sugawara H, Iwamoto K, Bundo M, Ueda J, Ishigooka J, Kato T. Comprehensive DNA methylation analysis of human peripheral blood leukocytes and lymphoblastoid cell lines. Epigenetics. 2011;6:508-15.

19. Åberg K, Khachane AN, Rudolf G, Nerella S, Fugman DA, Tischfield JA, van den Oord EJ. Methylome-wide comparison of human genomic DNA extracted from whole blood and from EBV-transformed lymphocyte cell lines. Eur J Hum Genet. 2012;20:953-5.

20. Thompson TM, Sharfi D, Lee M, Yrigollen CM, Naumova OY, Grigorenko EL. Comparison of whole-genome DNA methylation patterns in whole blood, saliva, and lymphoblastoid cell lines. Behav Genet. 2013;43:168-76.

21. Weber M, Hellmann I, Stadler MB, Ramos L, Pääbo S, Rebhan M, Schübeler D. Distribution, silencing potential and evolutionary impact of promoter DNA methylation in the human genome. Nat Genet. 2007:39:457-66.

22. Koga Y, Pelizzola M, Cheng E, Krauthammer M, Sznol M, Ariyan S, Narayan D, Molinaro AM, Halaban R, Weissman SM. Genome-wide screen of promoter methylation identifies novel markers in melanoma. Genome Res. 2009;19:1462-70.

23. Breitling LP, Yang R, Korn B, Burwinkel B, Brenner H. Tobacco-smokingrelated differential DNA methylation: $27 \mathrm{~K}$ discovery and replication. Am J Hum Genet. 2011;88:450-7.

24. Wan ES, Qiu W, Baccarelli A, Carey VJ, Bacherman H, Rennard SI, Agusti A, Anderson W, Lomas DA, Demeo DL. Cigarette smoking behaviors and time since quitting are associated with differential DNA methylation across the human genome. Hum Mol Genet. 2012;21:3073-82.

\section{Submit your next manuscript to BioMed Central and we will help you at every step:}

- We accept pre-submission inquiries

- Our selector tool helps you to find the most relevant journal

- We provide round the clock customer support

- Convenient online submission

- Thorough peer review

- Inclusion in PubMed and all major indexing services

- Maximum visibility for your research

Submit your manuscript at www.biomedcentral.com/submit
C Biomed Central 